\title{
Protecting Effects of Gonadotropin-Releasing Hormone Agonist on Chemotherapy-Induced Ovarian Damage in Premenopausal Breast Cancer Patients: A Systematic Review and Meta-Analysis
}

\author{
Fang Bai ${ }^{a}$ Yunshu Lu ${ }^{a}$ Kejin Wu ${ }^{b}$ Qing Chen ${ }^{a}$ Longlong Ding ${ }^{a}$ Meixin Ge ${ }^{a}$ Ziyi Weng $^{c}$ \\ a Department of General Surgery, Xinhua Hospital, Affiliated to Shanghai Jiao Tong University, School of Medicine, Shanghai, China; \\ ${ }^{b}$ Department of Breast Surgery, Gynecology and Obstetrics Hospital of Fudan University, Shanghai, China; \\ c Department of General Surgery, Shanghai International Medical Center, Shanghai, China
}

\begin{abstract}
Keywords
Premenopausal breast cancer - GnRH agonist .

Resumption of menses rate $\cdot$ Meta-analysis
\end{abstract}

\section{Summary}

Objective: There is no universal consensus on whether gonadotropin-releasing hormone $(\mathrm{GnRH})$ agonist could protect chemotherapy-induced ovarian damage in premenopausal breast cancer patients. This meta-analysis was conducted to estimate the protective effects of $\mathrm{GnRH}$ agonist on premenopausal breast cancer patients in details. Methods: PubMed, Cochrane Library, Embase, CNKI and the Chinese Wangfang Database, conference proceedings and clinical trials were searched to find studies reported since 2000 . Heterogeneity for the eligible data was assessed and a pooled odds ratio (OR) with 95\% confidence interval (Cl) was calculated. Results: Resumption of menses rate was improved in the $\mathrm{GnRH}$ agonist and chemotherapy-combination groups versus chemotherapy-alone groups (OR $=1.36,95 \% \mathrm{Cl}: 1.19$ 1.56). Furthermore, the results indicated that spontaneous pregnancy rate was improved in the experimental groups versus the controls $(\mathrm{OR}=1.90,95 \% \mathrm{Cl}: 1.06-$ 3.41). In addition, no publication bias was found using a Begg's funnel plot. Conclusion: The results of the current meta-analysis indicate that a $\mathrm{GnRH}$ agonist could im-

Fang Bai and Yunshu Lu have contributed equally to this work.

Ziyi Weng is co-corresponding author: Department of General Surgery, Shanghai International Medical Center, No. 4358 Kangxin Hwy. Pudong New District, Shanghai 201318, China; wzysn88@163.com prove resumption of menses rate and pregnancy rate for premenopausal breast cancer patients. However, more evaluation may be considered to prove this theory.

(c) 2017 S. Karger GmbH, Freiburg

\section{Introduction}

According to the updated data of GLOBOCAN, there were $1,677,000$ new cases of, and 522,000 deaths from, breast cancer worldwide in 2012, making it the most frequent type of cancer among women [1]. Chemotherapy has reduced the breast cancer death rate for breast cancer patients [2]. However, up to $60 \%$ of women treated with chemotherapy will experience periods of ovarian damage, which presents with a variety of symptoms, such as transient or permanent amenorrhea, infertility and early menopause, even premature ovarian failure (POF) and infertility. Each of these results in a significant financial and psycho-social burden [3-6]. As a consequence, preservation of ovarian function following adjuvant chemotherapy has raised great concern for both patients and clinicians/scientists. Fortunately, preclinical data have confirmed that temporary ovarian suppression with gonadotropinreleasing hormone $(\mathrm{GnRH})$ agonist during chemotherapy reduces ovarian toxicity through mechanisms such as interruption of follicle-stimulating hormone (FSH) secretion, a decrease in utero-ovarian perfusion, the activation of GnRH receptors on the oocytes, the up-regulation of intragonadal-anti-apoptotic molecules and/or the protection of undifferentiated germ-line stem cells [7, 8]. However, from the clinical trials, it still remains controversial as to whether

\section{KARGER}

() 2017 S. Karger GmbH, Freiburg

Fax +497614520714 
GnRH agonist can increase resumption of menses rate and pregnancy rate of premenopausal breast cancer patients. To help clarify this issue, we present a meta-analysis of 15 studies to give a quantitative assessment of the efficacy of GnRH agonists in protecting ovarian function in premenopausal breast cancer patients.

\section{Materials and Methods}

\section{Search Strategy}

Two investigators (F.B. and Y.L.) independently conducted a computerized search of all studies published since 2000 using the following databases: PubMed, Embase, Cochrane Library, CNKI and the Chinese Wangfang Database. In addition, we searched conference proceedings through online websites at www.asco.org and clinical trials through www.clinicaltrials.gov. A combination of Medical Subject Headings (MeSH) and text words was used to search the literature; these included gonadotropin-releasing hormone agonists, luteinizing hormone-releasing hormone agonists, triptorelin, goserelin, chemotherapy, ovarian preservation, premature ovary failure and premenopausal breast can-

Table 1. Check list for quality assessment

\begin{tabular}{|c|c|}
\hline Items & Score \\
\hline \multirow[t]{3}{*}{ Randomization } & $\begin{aligned} 0= & \text { not randomized in inappropriate method of } \\
& \text { randomization }\end{aligned}$ \\
\hline & $1=$ study described as randomized \\
\hline & $2=$ method of randomization described and appropriate \\
\hline \multirow{3}{*}{$\begin{array}{l}\text { Concealment of } \\
\text { allocation }\end{array}$} & $0=$ method of allocation concealment not described \\
\hline & $\begin{aligned} 1= & \text { study described as using allocation concealment } \\
& \text { method }\end{aligned}$ \\
\hline & $\begin{aligned} 2= & \text { method of allocation concealment described } \\
& \text { appropriately }\end{aligned}$ \\
\hline \multirow[t]{3}{*}{ Double blinding } & $0=$ not blind or inappropriate method of blinding \\
\hline & $1=$ study described as double blind \\
\hline & $2=$ method of double blind described and appropriate \\
\hline \multirow{2}{*}{$\begin{array}{l}\text { Withdrawals and } \\
\text { dropouts }\end{array}$} & $0=$ not described as follow-up \\
\hline & $1=$ description of withdrawals and dropouts \\
\hline
\end{tabular}

cer. We scanned the titles and abstracts to exclude any clearly irrelevant studies. The full texts of the remaining articles were reviewed to determine whether they contained information on the topic. In addition, the reference lists of the retrieved articles were checked to identify additional relevant publications.

\section{Inclusion Criteria}

The inclusion criteria were as follows: (1) the articles were published in English or Chinese; (2) the researches were restricted to those conducted on humans; (3) all the studies should have clear research objectives, designs and statistic methods; (4) the research subjects for the studies were female premenopausal breast cancer patients; (5) all of the studies should at least provide the statistics on the rate of resumption of menses, or it could be calculated; (6) the treatment of experimental group in all the included studies was a combination of $\mathrm{GnRH}$ agonist and chemotherapy, with the control group receiving chemotherapy therapy; and (7) the studies had to be randomized controlled trials (RCTs).

\section{Data Extraction}

Two independent reviewers (F.B. and Y.L.) extracted data from each eligible trial, including: (1) basic information, e.g. the year of publication, the name of first author and country of study; (2) study information, e.g. study population and type of study; (3) treatment information, e.g. regimen of both groups (dose, the time of administration, cycle and use of tamoxifen or radiation); and (4) other information, outcome measurement.

\section{Quality Assessment of Included Studies}

The quality of studies was assessed independently by 2 reviewers (F.B. and Y.L.) using the Modified Jadad Score [9]. In the assessment system, the quality of the studies was judged through 4 items: randomization, concealment of allocation, doubling blinding, and withdrawals and dropouts (table 1). The full score was 7 , and high-quality studies were defined by a score of $4-7$ (table 2).

\section{Statistical Analysis}

Data were collected and checked, and a database was set up as required for meta-analysis. The systematic assessment was performed using RevMan (Version 5.2). The comparison of the odds ratio (OR) of the rate of resumption of menses between experimental groups versus the control group in premenopausal breast cancer patients were the primary endpoints. Secondary endpoints were spontaneous pregnancy rate, and rate of resumption of menses among patients younger than 35 and for older patients. All the comparisons were calculated using the method for dichotomous data (assessment of OR; 95\% confidence interval (CI)). Both the fixed-effect model/Mantel-Haenszel method with minimal heterogeneity in the variables among studies and the Der SimonianLaird method (random effects model) when there was significant heterogeneity
Table 2. Quality assessments of studies

\begin{tabular}{llllll}
\hline Study & Randomization & $\begin{array}{l}\text { Concealment of } \\
\text { allocation }\end{array}$ & $\begin{array}{l}\text { Double } \\
\text { blinding }\end{array}$ & $\begin{array}{l}\text { Withdrawals } \\
\text { and dropouts }\end{array}$ & Total \\
\hline Badawy et al. [10] & 2 & 1 & 1 & 1 & 5 \\
Munster et al. [15] & 2 & 1 & 1 & 1 & 5 \\
Sverrisdottir et al. [13] & 2 & 1 & 1 & 1 & 5 \\
Del Mastro et al. [11] & 2 & 1 & 1 & 1 & 5 \\
Zhao et al. [20] & 2 & 1 & 0 & 1 & 4 \\
Gerber et al. [14] & 2 & 0 & 0 & 1 & 3 \\
Elgindy et al. [12] & 2 & 0 & 0 & 1 & 3 \\
Sun et al. [19] & 1 & 0 & 0 & 1 & 2 \\
Li et al. [18] & 1 & 0 & 0 & 1 & 2 \\
Jiang et al. [17] & 1 & 0 & 0 & 1 & 2 \\
Gilani et al. [16] & 1 & 0 & 0 & 1 & 2 \\
Lambertini et al. [23] & 2 & 1 & 0 & 1 & 4 \\
Song et al. [22] & 2 & 1 & 0 & 1 & 4 \\
Moore et al. [21] & 2 & 1 & 1 & 1 & 5 \\
Zhang et al. [24] & 2 & 0 & 0 & 1 & 3 \\
\hline
\end{tabular}


were used. The Cochran's Q test, with a predefined significance $\mathrm{p}$ threshold of 0.05 , was used to assess the statistical heterogeneity among the studies. The assumption of homogeneity was considered invalid for $\mathrm{p}$ values more than 0.05 . Funnel plots was used to test for possible publication bias. The presence of asymmetry in the funnel plot indicates the occurrence of publication bias.

\section{Results}

\section{Search Results}

The study selection is presented as a flowchart in figure 1. 186 records were searched in the database. Of these, 162 studies were

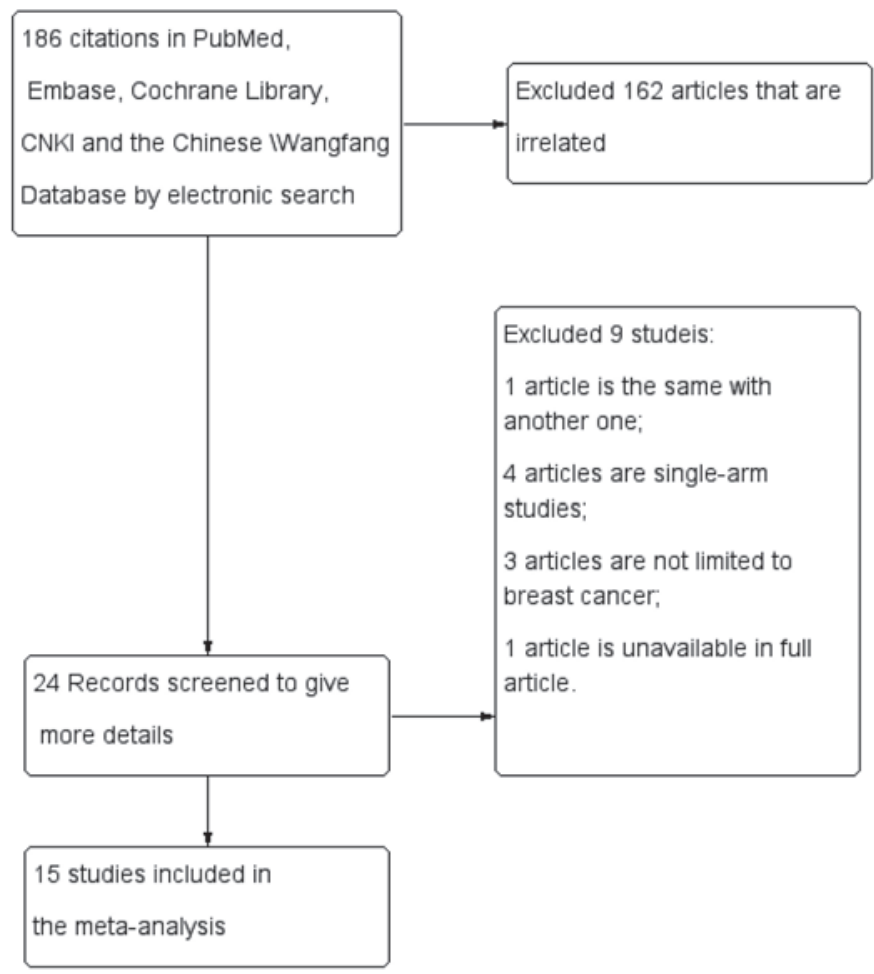

Fig. 1. Flow diagram. excluded after screening the title and abstract. Of the remaining 24, 15 records were included after full-text screening according to the inclusion criteria [10-24].

\section{Quality Evaluation and Study Characteristics}

Baseline characteristics of the 15 included studies are shown in online supplemental table 1 (www.karger.com/?DOI=454983). These studies were published between 2000 and 2015. Overall, 1,540 female premenopausal breast cancer patients were involved, and 786 of whom were in the experimental group, and 754 in the control group. All the studies were RCTs and were assessed through the Modified Jadad Score.

\section{Meta-Analysis}

According to the meta-analysis of the primary endpoint, there was between-study heterogeneity in the rate of resumption of menses (heterogeneity $\mathrm{Tau}^{2}=0.04, \mathrm{Chi}^{2}=51.98$ (d.f. $=14$ ), $\mathrm{I}^{2}=73 \%$, $\mathrm{p}<0.00001$ ). Therefore, we used the random-effect model to analyze the data and found that the rate of resumption of menses was improved in the experimental groups versus the controls $(\mathrm{OR}=1.36,95 \%$ CI: 1.19-1.56) (fig. 2).

As to the secondary endpoints, 5 studies $[11,14,15,21,23]$ were included and there was no between-study heterogeneity in spontaneous pregnancy rate (heterogeneity $\mathrm{Chi}^{2}=3.25$ (d.f. $=4$ ), $\mathrm{I}^{2}=0 \%$, $\mathrm{p}=0.52$ ). Therefore, we used the fixed-effect model, and the results indicated that spontaneous pregnancy was improved in the experimental groups versus the controls $(\mathrm{OR}=1.90,95 \% \mathrm{CI}: 1.06-3.41)$ (fig. 3).

In a subgroup analysis, we included 4 studies $[18-20,22]$ to compare the rate of resumption of menses among patients younger than 35 years, and there was no between-study heterogeneity (heterogeneity (d.f. $\left.=4), I^{2}=0 \%, p=0.47\right)$. Using the fixed-effect model to analyze the data, we found that the difference between 2 groups was not significant ( $\mathrm{OR}=0.69,95 \% \mathrm{CI}: 0.27-1.74)$. As to the rate of resumption of menses among patients older than 35 , there was between-study heterogeneity (heterogeneity (d.f. $=4$ ),
Fig. 2. Forest plot of rate of resumption of menses.

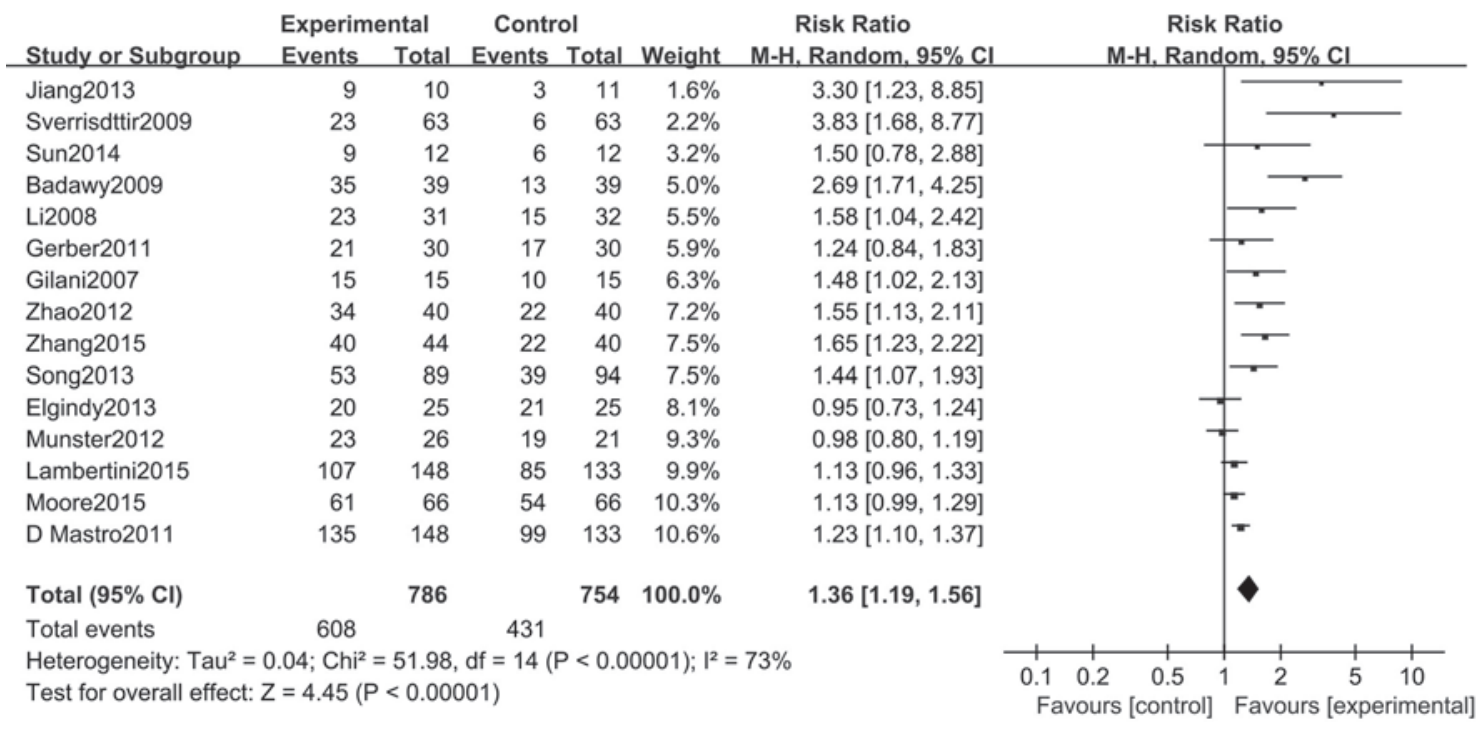

Bai/Lu/Wu/Chen/Ding/Ge/Weng 
Table 3. Subgroup analyses

\begin{tabular}{llllll}
\hline Patient age & Studies & \multicolumn{2}{l}{ Random effect } & \multirow{2}{*}{$\begin{array}{l}\text { Heterogeneity } \\
\text { p-value }\end{array}$} & I-squared, \% \\
\cline { 2 - 3 } & & OR $(95 \% \mathrm{CI})$ & p-Value & & \\
\hline$\leq 35$ years & 4 & $0.69(0.27,1.74)$ & 0.43 & 0.47 & 0 \\
$>35$ years & 4 & $3.22(0.56,18.65)$ & 0.19 & 0.0005 & 83 \\
\hline
\end{tabular}

*Included studies: Li et al. 2013 [18], Song et al. 2013 [22], Sun et al. 2014 [19], Zhao and Jia 2012 [20].

Fig. 3. Forest plot of pregnancy rate.

\begin{tabular}{|c|c|c|c|c|c|c|c|c|c|c|}
\hline Study or Subgroup & $\begin{array}{l}\text { Experim } \\
\text { Events }\end{array}$ & $\begin{array}{l}\text { ntal } \\
\text { Total }\end{array}$ & $\begin{array}{l}\text { Contr } \\
\text { Events }\end{array}$ & Total & Weight & $\begin{array}{l}\text { Odds Ratio } \\
\text { M-H. Fixed, } 95 \% \mathrm{Cl}\end{array}$ & & $\begin{array}{r}\text { Odds } \\
\text { M-H, Fixe }\end{array}$ & $\begin{array}{l}\text { s Ratio } \\
\text { ed. } 95 \% \mathrm{Cl}\end{array}$ & \\
\hline D Mastro2011 & 3 & 148 & 1 & 133 & $6.1 \%$ & $2.73[0.28,26.58]$ & & & & \\
\hline Gerber2011 & 1 & 30 & 1 & 30 & $5.7 \%$ & $1.00[0.06,16.76]$ & & & & \\
\hline Lambertini2015 & 8 & 148 & 3 & 133 & $17.8 \%$ & $2.48[0.64,9.53]$ & & & & \\
\hline Moore2015 & 22 & 105 & 12 & 113 & $54.3 \%$ & $2.23[1.04,4.77]$ & & & & \\
\hline Munster2012 & 0 & 26 & 2 & 21 & $16.1 \%$ & $0.15[0.01,3.24]$ & $\hookleftarrow$ & & & \\
\hline Total $(95 \% \mathrm{Cl})$ & & 457 & & 430 & $100.0 \%$ & $1.90[1.06,3.41]$ & & & & \\
\hline Total events & 34 & & 19 & & & & & & & \\
\hline \multicolumn{7}{|c|}{$\begin{array}{l}\text { Heterogeneity: } \mathrm{Chi}^{2}=3.25, \mathrm{df}=4(\mathrm{P}=0.52) ;\left.\right|^{2}=0 \% \\
\text { Test for overall effect: } Z=2.15(P=0.03)\end{array}$} & $\begin{array}{r}0.10 .2 \\
\text { Favol }\end{array}$ & $\begin{array}{l}0.5 \\
\text { [control] }\end{array}$ & $\begin{array}{l}1 \\
\text { Favours }\end{array}$ & $\begin{array}{cc}5 & 10 \\
\text { kperimental }\end{array}$ \\
\hline
\end{tabular}

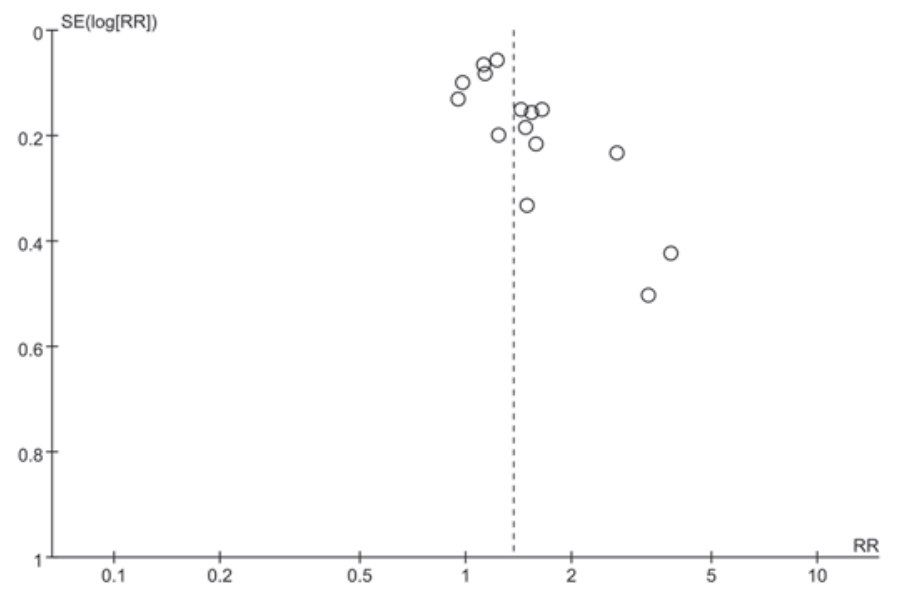

Fig. 4. Funnel plot of rate of resumption of menses.

$\left.\mathrm{I}^{2}=83 \%, \mathrm{p}=0.0005\right)$; using the random-effect model the difference between 2 groups was not significant $(\mathrm{OR}=3.22$, 95\% CI: $0.56-$ 18.65) (table 3).

\section{Publication Bias}

We performed the funnel plots to assess the publication bias. As a result, there was no publication bias in each for the primary endpoint analysis and the spontaneous pregnancy rate analysis (figs. 4 and 5).

\section{Discussion}

We included 15 studies in this meta-analysis, and the results indicate that GnRH agonist can significantly increase the rate of resumption menses. Subgroup comparison showed that the rate also increases in the patients younger than 35 years. In addition, the data prove that the spontaneous pregnancy rate improves significantly compared to control groups.

GnRH Agonist and Ovarian Damage MetaAnalysis

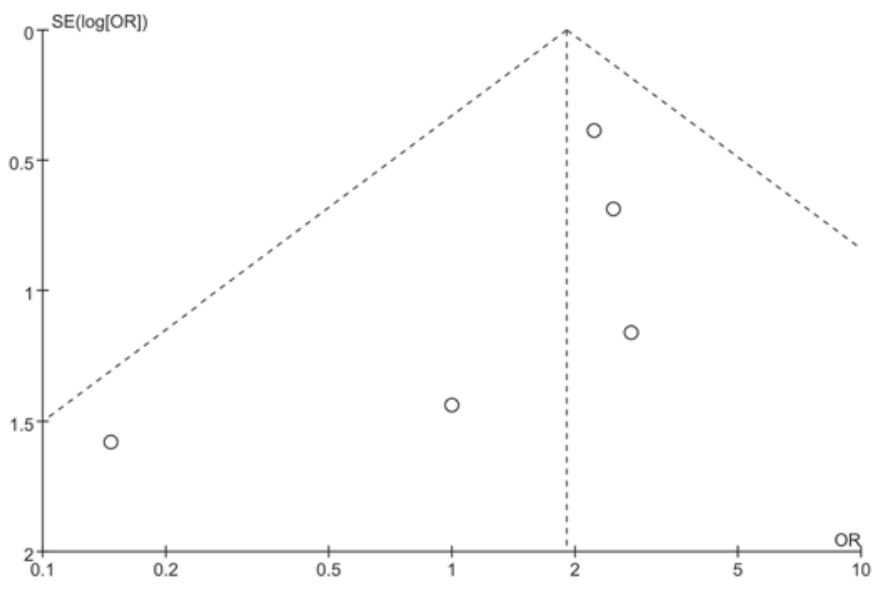

Fig. 5. Funnel plot of pregnancy rate.

The fundamental reason why GnRH agonists can provide ovarian protection is that prepubescent children have different rates of infertility after treatment with chemotherapy, with the prepubescent state seeming to confer some protection to the female gonads. A possible mechanism is that a $\mathrm{GnRH}$ agonist-induced hypoestrogenic state could decrease ovarian perfusion and that the $\mathrm{GnRH}$ agonist-induced hypogonadotropic milieu could decrease the number of primordial follicles entering the differentiation stage. In addition, ovarian cell apoptosis is decreased through either activation of the GnRH receptors or up-regulation of intragonadal antiapoptotic molecules. Furthermore, GnRH agonist may have certain protective effect on ovarian germline stem cells [25].

According to the NCCN Clinical Practice Guidelines in Oncology: breast cancer, version I 2016, randomized trials have shown that ovarian suppression with GnRH agonist therapy administered during adjuvant chemotherapy in premenopausal women with estrogen receptor-negative tumors may preserve ovarian function and diminish the likelihood of chemotherapy-induced amenorrhea 
[26]. Although resumption of menses is a clinically relevant and reproducible outcome, it is not necessarily linked to fertility restoration. Conversely, the presence of menses does not guarantee fertility. However, although ovarian suppression with GnRH agonist during chemotherapy has been studied as a strategy to preserve ovarian function and not fertility, there is evidence suggesting a possible utility of this technique to preserve fertility [27].

However, this meta-analysis still suffers from several limitations. First of all, different definitions of POF were used in different trials. Secondly, the limited length of follow-up in the RCTs precludes our ability to determine the long-term impact of GnRH agonists on preservation of ovarian function and fertility. Thirdly, because of lacking data, we were unable to compare the survival rates and hormones levels between patients with or without the use of GnRH agonist. Lastly, there was moderate heterogeneity among all included studies, for instance, the lack of uniform chemotherapy regimens.

In conclusion, GnRH agonists could be beneficial to premenopausal breast cancer patients by increasing the rates of resumption menses and pregnancy. To investigate this further, more research and high-quality homogeneous prospective clinical RCTs with larger populations and long-term follow-up are required.

\section{Online Supplemental Material}

Supplemental Table 1. Characteristics of included studies

To access the online supplemental table please refer to www.karger. $\mathrm{com} /$ ?DOI $=454983$.

\section{Acknowledgments}

The research was funded by Science and Technology Commission of Shanghai Municipality (No. 12JC1406800 and No. 14411950206).

\section{Disclosure Statement}

All authors declare that they have no financial or personal relationships with other people or organizations that could inappropriately influence their work.

\section{References}

1 Ferlay J, Soerjomataram I, Dikshit R, et al.: Cancer incidence and mortality worldwide: Sources, methods and major patterns in GLOBOCAN 2012. Int J Cancer 2015;136:E359-386.

2 Early Breast Cancer Trialists' Collaborative Group (EBCTCG): Effects of chemotherapy and hormonal therapy for early breast cancer on recurrence and 15year survival: An overview of the randomised trials. Lancet 2005;365:1687-1717.

3 Tiong V, Rozita AM, Taib NA, et al.: Incidence of chemotherapy-induced ovarian failure in premenopausal women undergoing chemotherapy for breast cancer. World J Surg 2014;38:2288-2296.

4 Ganz PA, Land SR, Geyer CE Jr, et al.: Menstrual history and quality-of-life outcomes in women with node-positive breast cancer treated with adjuvant therapy on the NSABPB-30 trial. J Clin Oncol 2011;29: 1110-1116.

5 Sivasubramaniam PG, Zhang BL, et al.: Breast cancer disparities: A multicenter comparison of tumor diagnosis, characteristics, and surgical treatment in China and the U.S. Oncologist 2015;20:1044-1050.

6 Sukumvanich P, Case LD, Van Zee K, et al.: Incidence and time course of bleeding after long-term amenorrhea after breast cancer treatment. Cancer 2011;116 3102-3111.

7 Blumenfeld Z, von Wolff M: GnRH analogues and oral contraceptives for fertility preservation in women during chemotherapy. Hum Reprod Update 2008;14:543552.

8 Clowse ME, Behera MA, Anders CK, et al.: Ovarian preservation by $\mathrm{GnRH}$ agonists during chemotherapy: A meta-analysis. J Womens Health (Larchmt) 2009;18: 311-319.

9 The Modified Jadad Scale for assessing the quality of studies. http://www.wenku.baidu.com/view/ 7b834a0c6c85ec3a87c2c5b1.html.
10 Badawy A, Elnashar A, El-Ashry M, Shahat M: Gonadotropin-releasing hormone agonists for prevention of chemotherapy-induced ovarian damage: Prospective randomized study. Fertil Steril 2009;91:694-697.

11 Del Mastro L, Boni L, Michelotti A, et al.: Effect of the gonadotropin-releasing hormone analogue triptorelin on the occurrence of chemotherapy-induced early menopause in premenopausal women with breast cancer a randomized trial. JAMA 2011;306:269-276.

12 Elgindy EA, El-Haieg DO, Khorshid OM, et al.: Gonadatrophin suppression to prevent chemotherapyinduced ovarian damage: A randomized controlled trial. Obstet Gynecol 2013;121:78-86.

13 Sverrisdottir A, Nystedt M, Johansson H, Fornander T: Adjuvant goserelin and ovarian preservation in chemotherapy treated patients with early breast cancer: Results from a randomized trial. Breast Cancer Res Treat 2009;117:561-567.

14 Gerber B, Minckwitz GV, Stehle H, et al.: Effect of luteinizing hormone releasing hormone agonist on ovarian function after modern adjuvant breast cancer chemotherapy: The GBG 37 ZORO study. J Clin Oncol 2011;29:2334-2341.

15 Munster PN, Moore AP, Ismail-Khan R, et al.: Randomized trial using gonadotropin-releasing hormone agonist triptorelin for the preservation of ovarian function during (neo)adjuvant chemotherapy for breast cancer. J Clin Oncol 2012;30:533-538.

16 Gilani MM, Hasanzadeh M, Ghaemmaghami F, Ramazanzadeh F: Ovarian preservation with gonadotropin-releasing hormone analog during chemotherapy. Asia Pac J Clin Oncol 2007;3:79-83.

17 Jiang FY, Zhang QQ, Zeng J: Protective effect of GnRH agonist on chemotherapy induced ovanan damage in breast cancer patients. Shandong Med J 2013;53:16-18.

18 Li MY, Huang H, Liang Y, Tan JM: Effect of zoladex administered before chemotherapy on menstruation of patients with breast cancer. Chin J Clin Oncol 2013;35: 905-907.
19 Sun JB, Liu L, Yang L, Ren YH. The clinical use of $\mathrm{GnRH}$ agonist to the resumption of menses of premenopausal breast cancer patients. Chin J Trauma Disability Med 2014;22:111-112.

20 Zhao XY, Jia GC: Ovarian protection with goserelin during adjuvant chemotherapy for pre-menopausal women with breast cancer. Zhengzhou University 2012;1-30.

21 Moore HC, Unger JM, Phillips KA, et al.: Goserelin for ovarian protection during breast-cancer adjuvant chemotherapy. N Engl J Med 2015;372:923-932.

22 Song G, Gao H, Yuan Z: Effect of leuprolide acetate on ovarian function after cyclophosphamide-doxorubicin-based chemotherapy in premenopausal patients with breast cancer: Results from a phase II randomized trial. Med Oncol 2013;30:667.

23 Lambertini M, Boni L, Michelotti A, et al.: Ovarian suppression with triptorelin during adjuvant breast cancer chemotherapy and long-term ovarian function, pregnancies, and disease-free survival: A randomized clinical trial. JAMA 2015;314:2632-2640.

24 Zhang L, Han L, Ma W: The effects of leuprolide acetate to breast cancer patients after chemotherapy. Chin J Clin Rational Drug Use 2015;8:121-123.

25 Blumenfeld $\mathrm{Z}$ : How to preserve fertility in young women exposed to chemotherapy? The role of $\mathrm{GnRH}$ agonist cotreatment in addition to cryopreservation of embrya, oocytes, or ovaries. Oncologist 2007;12:10441054.

26 National Comprehensive Cancer Network: NCCN Clinical Practice Guidelines in Oncology: breast cancer, version I 2016. http://www.nccn.org/professionals/ physician_gls/f_guidelines.asp.

27 Wong M, O’Neill S, Walsh G, Smith IE: Goserelin with chemotherapy to preserve ovarian function in premenopausal women with early breast cancer: Menstruation and pregnancy outcomes. Ann Oncol 2013;24: 133-138. 